INRA Prod. Anim., 2007, 20 (3), 223-226

\title{
Application de la modélisation en santé des espèces aquacoles
}

\author{
A. THEBAULT I', E.-J. PEELER ${ }^{2}$, A-G. MURRAY 3 , E. BRUN ${ }^{4}$, A. GIOVANINNI ${ }^{5}$, M-A. THRUSH ${ }^{6}$, \\ ${ }^{1}$ AFSSA, DERNS-PASER-AQR, 27-31 avenue du Général Leclerc, \\ F-94701 Maisons-Alfort, France \\ 2 EpiCentre, Institute of Veterinary and Animal Biomedical Sciences, Massey University, \\ Palmerston North, Nouvelle-Zélande \\ ${ }^{3}$ Fisheries Research Services, Marine Laboratory, 375 Victoria Road, \\ Aberdeen, AB11 9DB, Royaume-Uni \\ ${ }^{4}$ National Veterinary Institute, 0033 Oslo, Norvège \\ ${ }^{5}$ Istituto Zooprofilattico Spermentale dell'Abruzzo e del Molise, 64100, Teramo, Italie \\ ${ }^{6}$ Centre for Environment, Fisheries and Aquaculture Science, Barrack Road, \\ Weymouth, DT4 8UB, Royaume-Uni \\ Courriel : a.thebault@afssa.fr
}

Les questions posées en santé des espèces aquacoles sont nombreuses. L'augmentation des transferts et la cohabitation entre l'aquaculture et la faune sauvage sont responsables d'un certain nombre de cas recensées d'épizooties graves. Le nombre d'espèces concernées est bien plus important que pour la faune terrestre des pays développées et les outils de gestion du risque bien plus limités. Dans un tel contexte, des outils prédictifs deviennent particulièrement pertinents. Les démarches en évaluation des risques ont aussi été développées en aquaculture, le plus souvent par une démarche qualitative. $\mathrm{Si}$ des approches quantitatives sont menées, il semble qu'en aquaculture, les possibilités en terme de modélisation sont encore peu exploitées, du fait d'une méconnaissance de leurs possibilités, de leur intérêt, et de la disponibilité des modélisateurs. En France particulièrement, les travaux menés en terme de modélisation en santé des espèces aquacoles sur le plan international sont mal connus, c'est pourquoi une revue bibliographique a été menée. Afin de faciliter l'analyse bibliographique de ces travaux, les études de modélisation ont été regroupés en trois catégories : celles concernant l'appréciation des risques, celles concernant l'étude analytique des maladies, et un troisième groupe concernant les approches spatiales, spatio-temporelles, écologiques ou économiques. Cette division a pour objectif de mettre en avant les applications de ces modèles. Pour simplifier, l'appréciation des risques relève de la prévention et de la précaution, le deuxième groupe relève de l'étude des maladies présentes dans le territoire, et le troisième groupe d'une mise en relation avec des facteurs parfois nombreux et complexes qui peuvent expliquer ou prédire les causes et les conséquences de certains phénomènes. Bien évidemment, des relations existent entre ces 3 catégories.

Cette analyse s'appuie en grande partie sur les travaux réalisés dans le cadre d'un projet européen (DIPNET) coordonné par l'IFREMER et le CEFAS (Centre for Environment Fisheries and Agriculture Science)

\section{1 / Les applications de l'ap- préciation (quantitative/ qualitative) des risques en santé des espèces aquacoles}

Les approches quantitatives publiées sont limitées et concernent toutes les poissons, et en particulier exclusivement les salmonidés (saumons), en eau douce et en eau de mer. La première étude quantitative concerne le risque d'introduction de Gyrodactylus salaris dans les rivières norvégiennes par la salmoniculture (Paisley et al 1999). Cette approche a été complétée en 2001 (Paisley 2001) puis étendue au risque d'introduction entre rivières, en fonction de la distance (Hogasen et Brun 2003). D'autres agents ont fait l'objet d'études quantitatives, comme Myxobolus cerebralis (Bruneau 2001). La dernière étude concerne le risque de transmission verticale de Renibacterium salmoninarum dans les écloseries (Bovo et al 2002). Des approches qualitatives ont été menées en plus grand nombre concernant par exemple le risque lié aux introductions, aux transferts, mais aussi concernant le risque lié aux maladies émergentes (Peeler et Thrush 2004, Peeler et al 2004). Le schéma conceptuel des approches qualitatives pourrait être utilisé judicieusement pour des approches quantitatives par la suite.

Le champ d'application de l'appréciation du risque est vaste et doit répondre à une question posée par le gestionnaire du risque. Si la première étape est de dire quelle est la probabilité qu'un risque existe, la seconde est de savoir comment limiter celle-ci. L'appréciation des risques fournit des indications précieuses qui peuvent être utilisées pour la surveillance, comme cela a été effectué pour la seule étude concernant les coquillages et non les poissons (Thebault 2001). Les études publiées montrent des faiblesses, ainsi l'impact économique et écologique n'est le plus souvent pas estimé (Peeler et al 2006). D'autre part, les études ne séparent pas ce qui est lié à de l'incertitude ou de la variabilité, et ne présentent pas d'étude de sensibilité. La transparence des approches, notamment qualitatives, peut parfois être limitée notamment par rapport aux approches quantitatives publiées. La formulation des hypothèses peut revêtir une part de subjectivité qui ne doit pas être occultée. On peut remarquer que globalement la démarche d'appréciation des risques en santé des espèces aquacoles est sous-utilisée, ce qui vaut encore plus pour les approches quantitatives. Or, on peut imaginer que cette démar- 
che peut être extrapolée à d'autres productions, notamment à la conchyliculture. Le manque de données ne devant pas constituer un frein à la démarche conceptuelle qui peut aussi servir à hiérarchiser l'effort de recherche. Les données recherchées peuvent être aussi diversifiées que la morbidité liée à un agent infectieux, la survie de celui-ci dans le milieu extérieur, la vitesse de nage d'un smolt infecté, ou la fréquence des transferts. Les données devant être acquises sont donc issues d'études épidémiologiques, expérimentales ou même socio-économiques. Enfin les approches bayesiennes permettent d'utiliser de l'information a priori.

\section{2 / Les applications de modélisation en santé des espèces aquacoles en vue de l'étude analytique des ma- ladies}

Lorsque la maladie est installée, les questions qui peuvent être soulevées sont : est-ce que la maladie va se maintenir, ou disparaître, est-ce que les espèces présentes dans le milieu peuvent servir de réservoir, les espèces sauvages peuvent elles disparaître, comment diminuer les effets de la maladie ou couper la chaîne de transmission? Les études épidémiologiques sont le premier outil pour comprendre à l'échelle des populations les mécanismes de causalité et les moyens de lutte contre les maladies infectieuses. Les modèles dynamiques de transmission de maladie, nécéssitent un certain nombre d'informations et de connaissances qui peuvent nécessiter un certain temps d'obtention. Globalement, les études épidémiologiques rigoureuses sont relativement peu utilisées pour la gestion du risque. Les modèles s'appliquant aux mammifères terrestres, sont issus de l'épidémiologie prédictive, de type SIR ou SIS, et ont été extrapolé avec succès dans le domaine de la pathologie des poissons (Ogut et al 2004, Lorenzen et al 1991, Murray et Gaughan 2003). Des modèles issus de l'écologie tiennent aussi compte d'agents parasitaires pour décrire les dynamiques de populations de faune aquatique sauvage. Cependant, ces modèles restent relativement simples et ne tiennent pas compte, par exemple, des coinfections. Dans la conchyliculture, les modèles de dynamiques de maladies sont construits sur la physiologie des coquillages et sur les caractéristiques de l'environnement, selon des principes tirés de modèles écologiques (Hofman et al 1995, Ford et al 1999, Calvo et al 2003). Ces modèles sont satisfaisants pour des animaux poïkilothermes, très dépendants dans leur physiologie de leur environnement. Ces modèles sont par contre très couteux en données, et donc ne sont utilisables à des fins de gestion du risque qu'au bout d'un certain temps. Les modèles physiologiques-pathologique-écologiques «coquillages» ont été obtenus après 10 ans d'acquisition de données et de recherche. Il est donc dommage que trop peu d'études épidémiologiques soient mises en place pour répondre à des problèmes plus immédiats de gestion du risque. Les modèles dynamiques deviennent de toutes façon indispensables pour répondre à des chaînes de causalité plus complexes, ou pour prédire de façon quantitative l'effet de tel ou telle mesure qui ne peut mettre mis en place ou observable de façon simple in situ, comme l'effet du changement climatique (Hofman et al 2001). Dans le domaine de la pathologie des coquillages l'utilisation de modèles d'épidémiologie prédictives, plus simples que ceux développés aux USA, permettraient de démarrer la modélisation des maladies infectieuses européennes. Dans le domaine de la pathologie des poissons, les modèles existants sont amenés à évoluer vers des modèles plus complexes de type éco-physiologiques. Dans tous les cas, le facteur limitant pour toutes ces travaux de modélisation, utiles pour la gestion du risque est le manque d'études rigoureuses en épidémiologie descriptive et analytique, et parfois le manque de données expérimentales ou de terrains de base, comme la survie d'un parasite dans le milieu extérieur.

\section{3 / Les applications de modélisation en santé des espèces aquacoles en vue de l'étude écologique ou éco- nomique des maladies}

Les systèmes d'information géographiques et la modélisation hydrodynamique sont bien étudiés en milieu marin, particulièrement en France, grâce aux travaux de l'IFREMER. Vu les interactions de la faune aquatique avec son environnement, l'intégration de données spatialisées dans la modélisation est assez naturelle, et a été utilisée dans le domaine de la pathologie des poissons et de façon limitée en pathologie des coquillages (White et al
1998, Murray et al 2003). Pourtant les applications dans le domaine de la surveillance des maladies sont assez évidentes. L'intégration de dynamiques de populations sauvages ou élevées dans l'étude de maladies a été surtout mené par des écologistes, et ne concerne pas forcément les maladies les plus importantes du point de vue de l'élevage. Les interactions entre faune sauvage et d'élevage, le nombre d'espèces présentes qui cohabitent à côté les unes des autres, les épizooties ayant contaminé la faune sauvage montrent l'enjeu de ne pas négliger le tissu de relations qui existent entre les différentes espèces présentes dans le milieu marin. La modélisation classique atteint cependant ses limites mais de nouvelles approches méthodologiques permettent d'envisager des applications plus étendues dans ce domaine. Là encore, il faut souligner le manque de connaissances sur les agents infectieux dans la faune sauvage, le faible nombre de dynamiques de populations connus sur la faune sauvage marine et le manque d'interactions entre écologistes et pathologistes marins. Les coûts économiques de la surveillance et des mesures de lutte font aussi défaut pour aider le gestionnaire de risque dans ces choix. Cependant, la situation s'améliore dans le domaine de la pathologie des salmonidés grâce à des études récentes menées en Angleterre notamment. La démarche n'est pas abordée pour l'instant en pathologie des coquillages. Ces approches peuvent compléter par exemple, un exemple d'appréciation quantitative des risques. Cela nécessite des collaborations plus étroites entre économistes et pathologistes, ce qui est aussi une démarche connue et appliquée par les épidémiologistes.

\section{Conclusion}

La revue bibliographique souligne les enjeux et l'intérêt des travaux de modélisation en santé des epèces aquacoles. En France, cela permet de souligner les lacunes dans un domaine où de multiples compétences sont nécessaires et peut être difficiles à réunir pour ce type de production animale. Par exemple, de nombreuses données environnementales sont acquises en milieu marin, sans que ces données soient mises à profit dans le domaine de la santé des coquillages. La complexité et le manque de données sont souvent considérés comme des obstacles majeurs à toute tentative de modélisation en santé des espèces aquacoles. C'est partiellement vrai, car le travail de modélisation per- 
met aussi de mettre en avant les données prioritaires à acquérir, d'autre part les modèles publiés aux USA dans le domaine de la pathologie des coquillages et les modèles salmonidés du nord de l'Europe ont pu être mené jusqu'au bout, et ont pu contribuer utilement à la gestion du risque sur place. L'épidémiologie et l'écologie sont des disciplines peu utilisées pour l'instant en pathologie aquacole, malgré leur intérêt pour l'étude des phénomènes à l'échelle des populations, ce qui est relativement paradoxal pour des popu- lations sauvages ou cultivées en bien plus grand nombre, en nombre d'unités, que pour l'élevage terrestre. Il faut souligner que pour les coquillages, le faible nombre d'études réalisées n'est pas justifié par l'importance économique et sanitaire de cette production.

\section{Références}

Bovo G., Hastein T., Hill B., LaPatra S., Michel C., Olesen N., Shchelkunov I., Storset A., Wolffrom T., Midtlying P.J., 2002. Workpackage 1 report: hazard identification for vertical transfer of fish disease agents. Fish egg trade. Report QLK2 - CT - 2002 - 01546-ISBN 82-91743-355 , VESO, 35p.

Bruneau N., 2001. A quantitative risk assessment for the introduction of Myxobolus cerebralis to Alberta, Canada through the importation of live salmonids. In: Risk analysis in aquatic animal health. OIE (Ed.), Paris, France, 41-50.

Calvo L., Dungan C., Roberson R., Burreson E., 2003. Systematic evaluation of factors controlling Perkinsus marinus transmission dynamics in lower Chesapeake Bay. Dis. Aquat. Organ., 56, 75-86.

Ford S.E., Powell E.N., Klinck J.M., Hofmann E.E., 1999. Modelling the MSX parasite in eastern oyster (Crassostrea virginica) populations. I. Model development, implementation, and verification. J. Shellfish Res., 18, 475-500.

Hofmann E.E., Powell E.N., 1995. Modelling diseased oyster populations. I. Modelling Perkinsus marinus infections in oysters. J. Shellfish Res., 14, 121-151.

Hofman E., Ford S., Powell E., Klinck J., 2001. Modeling studies of the effect of climate variability on MSX disease in eastern oyster (Crassostrea virginica) population. Hydro-biologia, 460, 195-212.
Hogasen H.R., Brun E., 2003. Risk of interriver transmission of Gyrodactylus salaris by migrating Atlantic salmon smolts, estimated by Monte Carlo simulation. Dis. Aquat. Organ., 57, 247-254

Lorenzen K., Clers S., Anders K., 1991. Population dynamics of lymphocystis disease in estuarine flounder, Platichtys flesus(L.). J. Fish Biol., 39, 577-587.

Murray A.G., Gaughan D.J., 2003. Using an age structured model to simulate the recovery of the australian pilchard (sardinops sagax) population following epidemic mass mortality. Fish. Res., 60, 2-3.

Murray A.G., O'Callaghan M., Jones B., 2003. A model of spatially evolving herpesvirus epidemics causing mass mortality in Australian pilchard Sardinops sagax. Dis. Aquat. Organ., $54,1-14$.

Ogut H., Reno P.W., Sampson D., 2004. A deterministic model for the dynamics of furunculosis in chinook salmon Oncorhynchus tshawytscha. Dis. Aquat. Organ., 62, 57-63.

Paisley L.G., 2001. A Monte Carlo simulation model for assessing the risk of the introduction of Gyrodactylus salaris to the Tana River, Norway: a second scenario. In: OIE (Ed.), Risk analysis in aquatic animal health. OIE, Paris, France, 185-192.

Paisley L.G., Karlsen E., Jarp J., Mo T.A., 1999. A Monte Carlo simulation model for asses- sing the risk of introduction of Gyrodactylus salaris to the Tana river, Norway. Dis. Aquat. Organ., 37, 145-152.

Peeler E.J., Thrush M.A., 2004. Qualitative analysis of the risk of introducing Gyrodactylus salaris into the United Kingdom. Dis. Aquat. Organ., 62, 103-113.

Peeler E.J., Gardiner R., Thrush M.A., 2004. Qualitative risk assessment of routes of transmission of the exotic fish parasite Gyrodactylus salaris between river catchments in England and Wales. Prev. Vet. Med., 64, 175-189.

Peeler E.J., Murray A.G., Thebault A., Brun E., Thrush M.A., Giovannini A., 2006. Risk assessment and predictive modelling-a review of their application in aquatic animal health In: VESO (Ed.). European Commission, http://www.dipnet.info/.

Thebault A., 2001. Certifying the french population of Crassostrea gigas free from exotic diseases : a risk analysis approach. In: Risk analysis in aquatic animal health. OIE (Ed.), Paris, France, 61-70.

White D.L., Bushek D., Porter D.E., Edwards A.N., 1998. Geographic Information Systems (GIS) and kriging : analysis of the spatial and temporal distributions of the oyster pathogen perkinsus marinus in a developed and an undeveloped estuary. J. Shellfish Res., 17, 1473-1476.

\section{Résumé}

La lutte contre la propagation des maladies infectieuses et les conséquences de celles-ci de manière préventive est particulièrement importante en santé des espèces aquacoles, du fait des difficultés d'éradication des maladies une fois qu'elles sont introduites, notamment en milieu marin. Par conséquent, la modélisation en tant qu'outil prédictif est particulièrement justifiée. Une revue bibliographique a été menée dans le cadre du projet européen DIPNET en vue de dresser l'inventaire des modèles utilisés dans le domaine de la santé des espèces aquacoles. L'appréciation quantitative des risques commence à se développer pour certaines maladies des salmonidés d'Europe du nord, visant à garantir les transferts, aider les programmes, surveiller et prévenir l'apparition de maladies émergentes. Les modèles dynamiques de maladies commencent à être utilisés pour la gestion du risque, pour les salmonidés et pour les coquillages (USA) mais sont, suivant leur complexité, coûteux en données et en temps d'élaboration. Les modèles dynamiques sont indispensables cependant, quand on veut tester prédictivement l'effet de telle ou telle mesure, dans un système complexe de causalité. Les études épidémiologiques rigoureuses sont rares en santé des espèces aquacoles, malgré leurs intérêts démontrés dans le domaine de la santé. Les modèles décrivant le phénomène d'un point de vue spatial, les conséquences écologiques et économiques sont également rares en santé des espèces aquacoles. Ceci est paradoxal compte tenu des fortes interactions entre l'hôte et son environnement dans le milieu aquatique, et compte tenu de l'intérêt pour le gestionnaire de risque. Ces approches nécessitent cependant un travail impliquant des compétences multidisciplinaires, qu'il n'est pas forcément facile de réunir pour les productions aquacoles. 


\begin{abstract}
Modelling applications for aquatic animal health

Prevention is essential in order to fight against disease propagation and their consequences, in particular because curative methods are less efficient in the open and marine environment. Modelling as a predictive tool is in this case particularly accurate. A bibliographic review of published models in aquatic health was made for the European project DIPNET. Quantitative risk assessment is made for some diseases of salmonids of north European countries, in order to protect transfers, to make risk-based surveillance, and to prevent from emerging diseases. Dynamic models have begun to be used for risk management, for salmonids and shellfish (USA) but are depending on their complexity, data- and time-consuming. Dynamic models are, however, necessary for evaluating the effects of measures predictively, with a complex system of causality. Classical rigorous epidemiologic studies are not numerous in aquatic health, however they are of particular interest for risk management of health problems. For aquatic animal health, there are few models that take into account spatial analysis, economic or ecologic consequences. This is surprising because of the strong well-known interactions between the host and environment in aquatic systems, and because of the interest for the risk manager. Modelling work needs multidisciplinary skills, which are sometimes difficult to share for aquatic productions.
\end{abstract}

THEBAULT A., PEELER E.-J., MURRAY A.-G., BRUN E., GIOVANINNI A., THRUSH M.-A., 2007. Applications de la modélisation en santé des espèces aquacoles. INRA Prod. Anim., 20, 223-226. 